\title{
Iron abundance in the solar photosphere. Application of a two-component model atmosphere
}

\author{
L. R. Bellot Rubio ${ }^{1}$ and J. M. Borrero ${ }^{2}$
}

\author{
1 Kiepenheuer-Institut für Sonnenphysik, Schöneckstr. 6, 79104, Freiburg, Germany \\ 2 Max-Planck Institut für Aeronomie, 37191, Katlenburg-Lindau, Germany
}

Received 4 March 2002 / Accepted 23 April 2002

\begin{abstract}
A realistic two-component model of the quiet Sun is used to determine the solar abundance of iron from the inversion of a number of Fe I and Fe II spectral lines for which accurate atomic parameters (oscillator strengths, central wavelengths, and collisional broadening cross sections) exist. From $33 \mathrm{Fe}$ I lines we infer an abundance of $A_{\mathrm{Fe}}=7.43 \pm 0.06$, whereas we estimate $A_{\mathrm{Fe}}=7.45 \pm 0.08$ from $10 \mathrm{Fe}$ II lines. These values are in excellent agreement with the results of analyses based on realistic 3D hydrodynamical simulations of the solar granulation, and imply a low photospheric iron abundance. We investigate the effects of convective motions and granular temperatures and conclude that both are important for reliable abundance determinations. For Fe I lines, the effects of convective motions can be simulated by using a microturbulent velocity of about $1 \mathrm{~km} \mathrm{~s}^{-1}$, whereas it is possible to account for temperature inhomogeneities by adopting an average temperature stratification which is cooler than the Holweger \& Müller model in the upper layers.
\end{abstract}

Key words. Sun: photosphere - line: profiles - Sun: abundances - stars: atmospheres - stars: abundances

\section{Introduction}

Chemical abundances in the sun have traditionally been obtained by comparing observed spectral lines with the results of spectral syntheses. Given our limited capabilities to model the solar photosphere, the analyses have concentrated on fitting the observed equivalent widths rather than the whole line profiles. By doing so, a great deal of information that may help constrain the elemental abundances is neglected. Moreover, it is customary to use semiempirical one-component model atmospheres for the computation of synthetic spectra. These models are unable to reproduce the asymmetrical shapes of the observed profiles, which can be a potential source of systematic errors in the derived abundances. Another source of uncertainty is the lack of accurate line transition parameters, in particular oscillator strengths and collisional broadening constants.

These problems have led to a long standing debate on the exact chemical composition of the sun. This is true even for iron, by far the most studied element and the one for which better atomic parameters exist. Only recently, the various estimates of the solar iron abundance seem to converge to the meteoritic value (e.g., Anstee et al. 1997; Grevesse \& Sauval 1999; Asplund et al. 2000; Allende Prieto et al. 2001). However, small discrepancies remain, with some authors favoring an abundance close to 7.44 and others supporting a higher value of about 7.50.

The convergence mentioned above is the result of important advances in atomic physics and the development of new

Send offprint requests to: L. R. Bellot Rubio, e-mail: lbellot@kis.uni-freiburg.de analysis techniques. During the last years, significant improvements in the treatment of collisional broadening have been achieved. The quantum mechanical theory of Anstee, Barklem \& O'Mara (see Barklem et al. 2000 and references therein) makes enhancement factors to the classical Unsöld formula no longer necessary. This removes a very important source of error. Another improvement is the gradual switch from equivalent width fitting to profile fitting. Inversion techniques, based on either one-component model atmospheres (Allende Prieto et al. 2001) or multi-component models (Frutiger et al. 2000), have played a crucial role in recent analyses. Finally, the advent of highly realistic 3D numerical simulations of the solar convection makes the determination of chemical abundances independent of parameters like microturbulence or macroturbulence. Again, this removes an important source of error. Remarkably, analyses based on one-component model atmospheres tend to favor high values of the iron abundance, while those employing the results of numerical simulations yield lower values. Very likely, the origin of such small differences is the inability of one-component models to reproduce the line asymmetries caused by convective motions. This has already been recognized by several authors (e.g. Grevesse \& Sauval 1999; Allende Prieto et al. 2001) as a fundamental limitation of one-component models of the solar photosphere.

In the present work we derive the photospheric iron abundance by using a realistic two-component model of the quiet sun. Our analysis is similar to those based on profile fitting techniques and semiempirical model atmospheres, the only difference being our using a more complex model which is 
able to describe the effects of convective motions in the solar photosphere. To our knowledge, this is the first time a well tested two-component model is applied to derive abundances in the sun. The results presented here help understand the influence of neglecting convective flows on current abundance determinations.

\section{Model atmospheres and spectral lines}

The two-component model atmosphere used in this work has been obtained by Borrero \& Bellot Rubio (2002) from a LTE inversion of $22 \mathrm{Fe} I$ lines. The model is meant to describe convective flows in the solar photosphere. Its two components can be regarded as idealized representations of granular and intergranular cells. In addition to mimicking the full shape of the spectral lines used in the inversion, the model is able to reproduce a wide range of observables of the solar intensity spectrum. These include the convective line shifts and equivalent widths of nearly a thousand lines (mainly iron lines, but also calcium, silicon, titanium, and chromium lines). The model is also capable of reproducing the observed center-to-limb variation of the continuum intensity with better accuracy than classical one-component atmospheres. Because of the higher degree of realism, it is clear that current abundance studies would benefit from the application of such a two-component model.

For reliable inferences of chemical abundances it is crucial to use spectral lines with very accurate atomic parameters. In addition, they should appear clean and have a well defined continuum in the solar spectrum. The lines selected for treatment are presented in Table 1. For the most part, they have been extracted from the compilations of Grevesse \& Sauval (1999) and Asplund et al. (2000, hereafter ANTS). Some Fe I lines in ANTS have not been included either because they were employed to obtain the two-component model atmosphere or because of problems with blends or continuum placement. Three additional Fe II lines in Grevesse \& Sauval (1999) have been removed for the same reasons. It is important to emphasize that the oscillator strengths of these lines have been measured in the laboratory by the Oxford and/or Hannover-Kiel groups. Central wavelengths for Fe I and Fe II lines are taken from Nave et al. (1994) and Johansson (as quoted by ANTS), respectively. We use experimental values for the radiative broadening (extracted from the VALD database, Kupka et al. 1999) and quantum mechanical cross-sections for collisional broadening by neutral hydrogen (Barklem et al. 2000 and references therein). We deem that these data are of the highest quality, but at the same time acknowledge the lower accuracy of atomic parameters for ionized iron. Our final list contains $33 \mathrm{Fe} \mathrm{I}$ and $10 \mathrm{Fe}$ II spectral lines. The reason for the small number of Fe II lines considered here is the paucity of clean lines in the solar spectrum, together with the lack of good atomic parameters.

The intensity profiles of the lines have been extracted from the spectral atlas of Brault \& Neckel (1987) (see also Neckel 1994), which gives the spatially and temporally averaged solar intensity at disk center from 3290 to $12510 \AA$. The data were taken with the Fourier Transform spectrometer (FTS) attached to the McMath/Pierce telescope at Kitt Peak. The wavelength calibration of the atlas has been shown to be free of systematic errors by Allende Prieto \& García López (1997). An additional advantage of these observations is that the instrumental profile of the FTS is very narrow. Thus, we do not need to consider it for the spectral synthesis. This would not be the case if a conventional spectrograph had been used. Prior to the analysis, the sections of the observed profiles affected by blends (including possible very weak ones) have been removed.

\section{Analysis}

Synthetic profiles have been computed by using the twocomponent model atmosphere and the atomic parameters listed in Table 1. The observed profiles have been fitted to the synthetic lines with the help of the SIR inversion code (Ruiz Cobo \& del Toro Iniesta 1992). The radiative transfer module of SIR is based on Wittmann (1974) routines, and so is the computation of continuum opacities. Local thermodynamical equilibrium (LTE) is assumed throughout. The only free parameters allowed to vary during the fit are the chemical abundance and the central wavelength of the line under consideration. To this end, the corresponding response functions (partial derivatives of the emergent intensity with respect to the free parameters) have been implemented in the inversion code. The fitting of the observed profile (by means of a $\chi^{2}$ minimization) is carried out automatically to ensure that the optimum solution is found. We emphasize that the line cores are not considered in the fit wherever the intensity drops below $0.35 I_{\mathrm{c}}$. This should help minimize departures from LTE, which are more likely to affect the core than the line wings.

An example of the quality of the inversions is given in Fig. 1, where the observed and best-fit profiles of two Fe I and Fe II lines are shown. Except for a few lines, the observed profiles are very satisfactorily reproduced, including their convective asymmetries. In general, Fe II lines yield poorer fits as compared with Fe I lines. This hints at small errors in the model, which was obtained from the inversion of neutral iron lines only. NLTE effects are the most likely source of such errors. Indeed, the different departures of neutral and ionized iron lines from LTE make it difficult or even impossible to find temperature stratifications capable of reproducing both Fe I and Fe II lines under strict LTE conditions. For a number of reasons, however, we believe that these errors have a minor impact on the derived abundances (see the discussion in Borrero \& Bellot Rubio 2002). Another explanation for the poorer fits is the generally lower quality of atomic parameters for Fe II lines. In any case, the fits are reasonably good and of course better than those based on one-component model atmospheres.

\section{Results}

The individual iron abundances derived from our twocomponent model are listed in Table 1 under the column $A(2 \mathrm{C})$. The average value for the $33 \mathrm{Fe}$ I lines is $A_{\mathrm{Fe}}=7.43 \pm 0.06$ in the usual logarithmic scale. From the $10 \mathrm{Fe}$ II lines we obtain an average abundance of $A_{\mathrm{Fe}}=7.45 \pm 0.08$. Both values agree within their uncertainties, although the scatter is much larger in the case of ionized iron. This clearly reflects the poorer quality of transition probabilities for Fe II lines. 
Table 1. Spectral lines selected for analysis. The different columns give the species, air central wavelength $\lambda_{0}$, excitation potential $\chi_{1}$, oscillator strength $\log g f$ and its source, temperature exponent $\alpha$ and cross section $\sigma_{0}$ (in units of $a_{0}^{2}$, with $a_{0}$ being Bohr's radius) for collisional broadening, radiative broadening $\log \gamma_{\text {rad }}$, and minimum intensity of the line in the FTS atlas. The next three columns indicate the abundances derived from the one-component model atmosphere of Holweger \& Müller (1974), from the two-component model of Borrero \& Bellot Rubio (2002), and from the 3D hydrodynamical simulations of ANTS, respectively. The ANTS abundances for Fe II lines have been rescaled to the Schnabel et al. (1999) oscillator strengths.

\begin{tabular}{|c|c|c|c|c|c|c|c|c|c|c|c|}
\hline Species & $\begin{array}{l}\lambda_{0}^{\mathrm{a}} \\
(\AA)\end{array}$ & $\begin{array}{c}\chi_{1} \\
(\mathrm{eV})\end{array}$ & $\begin{array}{c}\log g f \\
(\operatorname{dex})\end{array}$ & Source $^{b}$ & $\alpha$ & $\begin{array}{c}\sigma_{0} \\
\left(a_{0}^{2}\right)\end{array}$ & $\begin{array}{c}\log \gamma_{\mathrm{rad}} \\
\left(\mathrm{s}^{-1}\right)\end{array}$ & $I_{\min }$ & $\begin{array}{c}A(1 \mathrm{C}) \\
(\mathrm{dex})\end{array}$ & $\begin{array}{c}A(2 \mathrm{C}) \\
(\mathrm{dex})\end{array}$ & $\begin{array}{c}A(3 \mathrm{D}) \\
(\mathrm{dex})\end{array}$ \\
\hline Fe I & 4389.2451 & 0.052 & -4.583 & $\mathrm{e}$ & 0.249 & 217 & 4.529 & 0.14 & 7.53 & 7.42 & 7.43 \\
\hline $\mathrm{Fe} \mathrm{I}$ & 4424.0683 & 3.642 & -2.312 & $\mathrm{a}$ & 0.248 & 330 & 8.158 & 0.67 & 7.49 & 7.46 & \\
\hline $\mathrm{Fe} \mathrm{I}$ & 4787.8269 & 2.997 & -2.530 & $\mathrm{~b}$ & 0.227 & 818 & 7.783 & 0.50 & 7.44 & 7.40 & \\
\hline $\mathrm{Fe} \mathrm{I}$ & 4924.7695 & 2.279 & -2.241 & $\mathrm{a}$ & 0.244 & 360 & 8.161 & 0.18 & 7.64 & 7.59 & \\
\hline $\mathrm{Fe} \mathrm{I}$ & 4939.6867 & 0.859 & -3.315 & $\mathrm{f}$ & 0.246 & 244 & 7.193 & 0.16 & 7.57 & 7.39 & \\
\hline $\mathrm{Fe} I$ & 5044.2114 & 2.851 & -2.059 & $\mathrm{~g}$ & 0.238 & 713 & 8.009 & 0.26 & 7.51 & 7.45 & 7.45 \\
\hline $\mathrm{Fe} \mathrm{I}$ & 5247.0503 & 0.087 & -4.946 & $\mathrm{e}$ & 0.253 & 206 & 3.894 & 0.25 & 7.52 & 7.38 & 7.42 \\
\hline $\mathrm{Fe} \mathrm{I}$ & 5250.2090 & 0.121 & -4.938 & $\mathrm{e}$ & 0.253 & 208 & 3.643 & 0.26 & 7.55 & 7.41 & 7.45 \\
\hline $\mathrm{Fe} \mathrm{I}$ & 5253.4619 & 3.283 & -1.573 & $\mathrm{~g}$ & 0.229 & 849 & 7.875 & 0.27 & 7.46 & 7.40 & 7.40 \\
\hline $\mathrm{Fe} \mathrm{I}$ & 5525.5444 & 4.230 & -1.084 & $\mathrm{~g}$ & 0.238 & 749 & 8.382 & 0.44 & 7.41 & 7.40 & 7.39 \\
\hline $\mathrm{Fe} \mathrm{I}$ & 5696.0896 & 4.548 & -1.720 & $\mathrm{a}$ & 0.279 & 965 & 8.279 & 0.86 & 7.37 & 7.35 & \\
\hline Fe I & 5701.5444 & 2.559 & -2.216 & e & 0.237 & 361 & 8.167 & 0.28 & 7.58 & 7.48 & 7.53 \\
\hline $\mathrm{Fe} \mathrm{I}$ & 5705.4648 & 4.301 & -1.355 & $\mathrm{~g}$ & 0.231 & 744 & 8.290 & 0.59 & 7.43 & 7.42 & 7.38 \\
\hline $\mathrm{Fe} \mathrm{I}$ & 5712.1316 & 3.417 & -1.990 & $\mathrm{~b}$ & 0.240 & 817 & 7.877 & 0.46 & 7.48 & 7.44 & \\
\hline $\mathrm{Fe} I$ & 6065.4822 & 2.608 & -1.530 & $\mathrm{~d}$ & 0.234 & 354 & 8.009 & 0.24 & 7.55 & 7.40 & \\
\hline $\mathrm{Fe} \mathrm{I}$ & 6136.9946 & 2.198 & -2.950 & $\mathrm{e}$ & 0.265 & 280 & 8.217 & 0.37 & 7.53 & 7.43 & 7.46 \\
\hline $\mathrm{Fe} \mathrm{I}$ & 6219.2808 & 2.198 & -2.433 & $\mathrm{e}$ & 0.264 & 278 & 8.190 & 0.29 & 7.43 & 7.34 & 7.45 \\
\hline $\mathrm{Fe} \mathrm{I}$ & 6229.2283 & 2.845 & -2.805 & $\mathrm{~b}$ & 0.250 & 350 & 8.161 & 0.62 & 7.42 & 7.36 & \\
\hline $\mathrm{Fe} \mathrm{I}$ & 6265.1338 & 2.176 & -2.550 & $\mathrm{e}$ & 0.261 & 274 & 8.220 & 0.30 & 7.50 & 7.38 & 7.45 \\
\hline $\mathrm{Fe} \mathrm{I}$ & 6270.2250 & 2.858 & -2.464 & $\mathrm{~b}$ & 0.249 & 350 & 8.161 & 0.48 & 7.41 & 7.35 & \\
\hline $\mathrm{Fe} \mathrm{I}$ & 6271.2788 & 3.332 & -2.703 & $\mathrm{~g}$ & 0.247 & 720 & 8.074 & 0.76 & 7.49 & 7.45 & 7.40 \\
\hline $\mathrm{Fe} \mathrm{I}$ & 6280.6182 & 0.859 & -4.387 & $\mathrm{e}$ & 0.253 & 223 & 4.622 & 0.37 & 7.59 & 7.45 & 7.46 \\
\hline Fe I & 6297.7930 & 2.223 & -2.740 & e & 0.264 & 278 & 8.190 & 0.34 & 7.50 & 7.40 & 7.44 \\
\hline $\mathrm{Fe} \mathrm{I}$ & 6311.5003 & 2.831 & -3.141 & $\mathrm{~b}$ & 0.250 & 344 & 8.161 & 0.71 & 7.56 & 7.51 & \\
\hline Fe I & 6322.6855 & 2.588 & -2.426 & $\mathrm{e}$ & 0.238 & 345 & 8.009 & 0.35 & 7.57 & 7.47 & 7.51 \\
\hline $\mathrm{Fe} \mathrm{I}$ & 6518.3671 & 2.831 & -2.450 & $\mathrm{a}$ & 0.251 & 336 & 8.167 & 0.46 & 7.44 & 7.38 & \\
\hline $\mathrm{Fe} \mathrm{I}$ & 6593.8706 & 2.433 & -2.422 & $\mathrm{e}$ & 0.247 & 321 & 7.936 & 0.34 & 7.57 & 7.48 & 7.53 \\
\hline $\mathrm{Fe} \mathrm{I}$ & 6609.1104 & 2.559 & -2.692 & $\mathrm{e}$ & 0.245 & 335 & 7.905 & 0.42 & 7.57 & 7.48 & 7.49 \\
\hline $\mathrm{Fe} \mathrm{I}$ & 6663.4421 & 2.424 & -2.453 & $\mathrm{~b}$ & 0.239 & 337 & 6.843 & 0.35 & 7.49 & 7.37 & \\
\hline $\mathrm{Fe} \mathrm{I}$ & 6978.8516 & 2.484 & -2.500 & e & 0.241 & 337 & 6.886 & 0.37 & 7.55 & 7.44 & 7.49 \\
\hline $\mathrm{Fe} \mathrm{I}$ & 7112.1720 & 2.990 & -2.998 & $\mathrm{~b}$ & 0.238 & 374 & 8.167 & 0.74 & 7.54 & 7.49 & \\
\hline $\mathrm{Fe} \mathrm{I}$ & 7189.1510 & 3.071 & -2.771 & g & 0.260 & 344 & 8.161 & 0.64 & 7.62 & 7.57 & \\
\hline Fe I & 7941.0892 & 3.274 & -2.286 & $\mathrm{~b}$ & 0.264 & 349 & 8.161 & 0.64 & 7.35 & 7.31 & \\
\hline $\mathrm{Fe}$ II & 4576.3334 & 2.844 & -2.90 & $\mathrm{c}$ & 0.267 & 188 & 8.612 & 0.29 & 7.41 & 7.45 & 7.38 \\
\hline $\mathrm{Fe}$ II & 4620.5129 & 2.828 & -3.19 & $\mathrm{c}$ & 0.267 & 188 & 8.615 & 0.39 & 7.32 & 7.34 & 7.32 \\
\hline $\mathrm{Fe}$ II & 4656.9762 & 2.891 & -3.57 & $\mathrm{c}$ & 0.330 & 190 & 8.612 & 0.56 & 7.39 & 7.40 & 7.38 \\
\hline Fe II & 5234.6243 & 3.221 & -2.22 & $\mathrm{c}$ & 0.268 & 188 & 8.487 & 0.26 & 7.64 & 7.56 & 7.48 \\
\hline $\mathrm{Fe}$ II & 5414.0717 & 3.221 & -3.48 & $\mathrm{c}$ & 0.268 & 188 & 8.615 & 0.70 & 7.42 & 7.38 & 7.36 \\
\hline Fe II & 6432.6757 & 2.891 & -3.51 & $\mathrm{c}$ & 0.270 & 188 & 8.462 & 0.70 & 7.45 & 7.41 & 7.39 \\
\hline $\mathrm{Fe}$ II & 6516.0716 & 2.891 & -3.38 & $\mathrm{c}$ & 0.270 & 174 & 8.464 & 0.53 & 7.60 & 7.57 & 7.52 \\
\hline Fe II & 7224.4790 & 3.889 & -3.28 & $\mathrm{c}$ & 0.275 & 188 & 8.617 & 0.83 & 7.62 & 7.55 & 7.55 \\
\hline $\mathrm{Fe}$ II & 7515.8309 & 3.903 & -3.37 & c & 0.271 & 187 & 8.612 & 0.88 & 7.56 & 7.44 & 7.43 \\
\hline $\mathrm{Fe}$ II & 7711.7205 & 3.903 & -2.45 & $\mathrm{c}$ & 0.264 & 186 & 8.615 & 0.64 & 7.43 & 7.40 & 7.39 \\
\hline
\end{tabular}

a Central wavelengths from Nave et al. (1994) for Fe I lines and Johansson (private communication) for Fe II lines.

b Sources of $f$-values: (a) Bard \& Kock (1994), (b) Bard et al. (1991), (c) Schnabel et al. (1999), (d) Blackwell et al. (1982), (e) Blackwell et al. (1995a), (f) Blackwell et al. (1995b), (g) Holweger et al. (1995).

For comparison purposes, the same abundance determination has been carried out adopting the one-component model of Holweger \& Müller (1974, hereafter HM) with a macroturbulent velocity of $1 \mathrm{~km} \mathrm{~s}^{-1}$. As it is usual in $1 \mathrm{D}$ abundance analyses, we have assumed a depth-independent microturbulence of $1 \mathrm{~km} \mathrm{~s}^{-1}$ in order to compensate for the neglect of convective motions. The individual abundances derived in this way are listed in Table 1 under the column $A(1 \mathrm{C})$. 

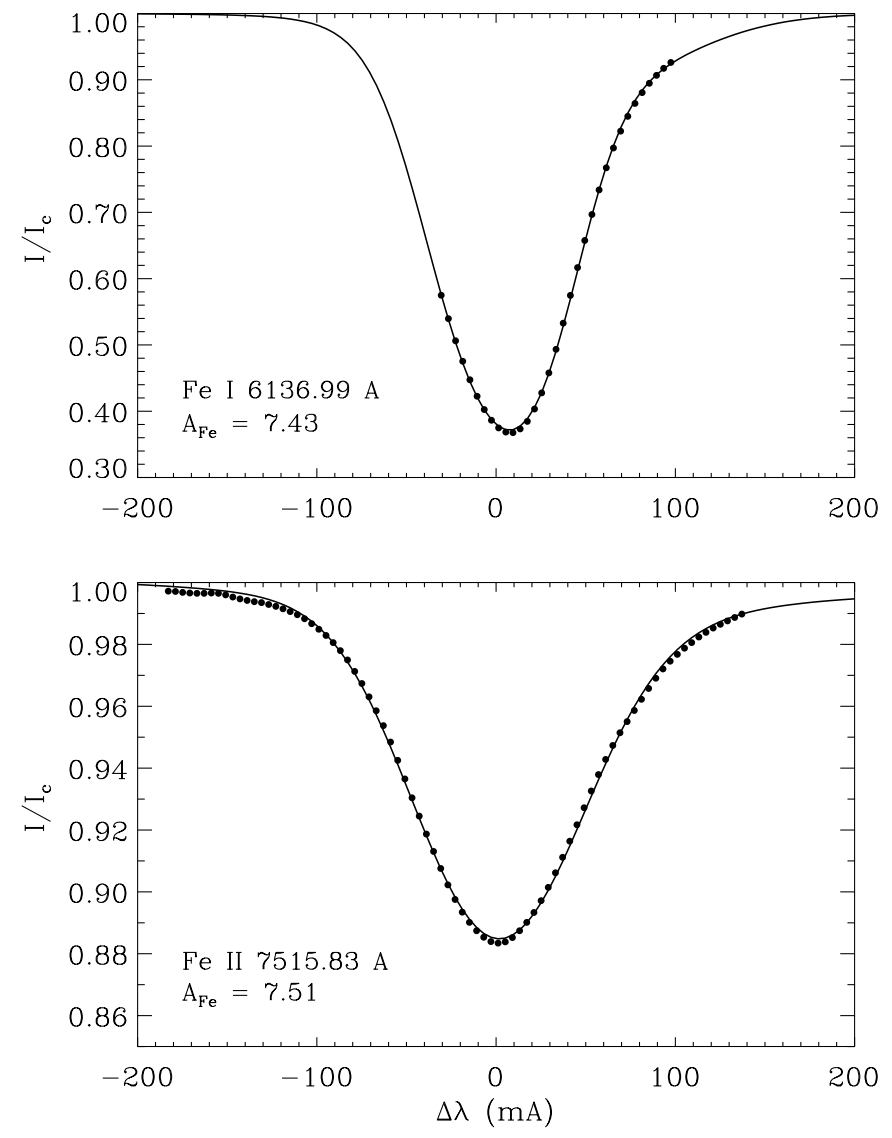

Fig. 1. Observed (filled circles) and best-fit (solid lines) profiles for the Fe I $6136.99 \AA$ and Fe II $7515.83 \AA$ lines.

The average iron abundance obtained with the HM model is $A_{\mathrm{Fe}}=7.50 \pm 0.07$ from Fe I lines and $A_{\mathrm{Fe}}=7.48 \pm 0.11$ from Fe II lines. These values agree remarkably well with those found in earlier investigations based on semiempirical one-component models. Allende Prieto et al. (2001), for example, obtained $A_{\mathrm{Fe}}=7.51 \pm 0.02$ from the simultaneous inversion of the flux profiles of $24 \mathrm{Fe}$ I and Fe II lines. Grevesse \& Sauval (1999) found $A_{\mathrm{Fe}}=7.50 \pm 0.05$ from fits to the equivalent widths of $65 \mathrm{Fe} \mathrm{I}$ and $13 \mathrm{Fe}$ II lines using a modified HM model. Our two-component results from Fe I lines are about 0.07 dex lower than these one-component abundances. This suggests that switching to more complex two-component model atmospheres (thus allowing for line asymmetries in the synthetic spectra) slightly decreases the derived abundances.

Also for comparison purposes, the individual iron abundances determined by ANTS from 3D numerical simulations of the solar granulation are given in Table 1 under the column $A(3 \mathrm{D})$. The average values from these $3 \mathrm{D}$ models are $A_{\mathrm{Fe}}=7.44 \pm 0.04$ (Fe I lines) and $A_{\mathrm{Fe}}=7.43 \pm 0.10$ (Fe II lines, rescaled to Schnabel et al. 1999 oscillator strengths). The iron abundance resulting from the application of the two-component model atmosphere is thus in excellent agreement with the values of ANTS, the only difference being our slightly larger scatter. Such larger scatter can be traced to the simplicity of the two-component model, which of course do not provide an exact representation of all individual atmospheres that contribute to the observed profiles. In spite of this, the similarity of the results suggests that simple semiempirical models incorporating a rough description of convective motions can be used to determine reliable abundance values. Among other advantages, they are much easier to construct and handle than 3D numerical models, so they are of interest when 3D simulations cannot be afforded.

As an additional test, we have checked the individual abundances for trends with wavelength, excitation potential and line strength. The results are shown in Fig. 2 for the values determined from the one-component HM model (first row), our two-component model atmosphere (second row), and the 3D hydrodynamical simulations of ANTS (third row). No significant dependences can be seen for the two-component model and the 3D simulations. However, the HM model results in a strong trend with excitation potential. Low excitation lines systematically yield larger abundances than high excitation lines. Grevesse \& Sauval (1999) already found this trend and showed that it can be removed by decreasing the temperature of the model in the upper layers. Our two-component analysis and the 3D hydrodynamical simulations indicate that a proper account of granular temperatures and velocities also leads to abundances which do not depend on the excitation potential of the lines.

\section{Discussion}

We have seen in the previous section that the iron abundance resulting from the application of a two-component model atmosphere is slightly lower than those based on one-component models. Since it agrees remarkably well with the values obtained from highly realistic 3D numerical simulations, the question naturally arises as to the origin of such a difference.

In order to gain more insight into this problem, we have repeated the same abundance determination using our twocomponent model with (a) the velocity fields artificially set to zero; (b) the velocity fields substituted by a microturbulence of $1 \mathrm{~km} \mathrm{~s}^{-1}$; and (c) the original velocity fields, but with the granular and intergranular temperatures substituted by the average temperature stratification. In this way we separate the effects of convective motions and temperature inhomogeneities. The results of the analysis are summarized in Fig. 3. Given the small number of Fe II lines and the large scatter of the abundances derived from them, we will restrict our discussion to the more numerous Fe I lines. When the velocity fields are removed from the two-component model (upper panels), the individual iron abundances show strong trends with excitation potential and line strength. These trends resemble those found upon application of the HM model (see upper panels in Fig. 2). The average iron abundance from Fe I lines is $A_{\mathrm{Fe}}=7.47 \pm 0.09$, i.e., midway between the value obtained from the HM model and the complete two-component model. When the velocity fields are substituted by a microturbulence of $1 \mathrm{~km} \mathrm{~s}^{-1}$ (central panels), the trends with excitation potential and line strength largely disappear and the scatter is reduced compared to the previous case. The mean iron abundance is found to be $A_{\mathrm{Fe}}=7.41 \pm 0.06$, i.e., slightly lower than that resulting from the complete twocomponent model. Finally, if the original velocity fields are kept but the temperatures of the granular and intergranular 

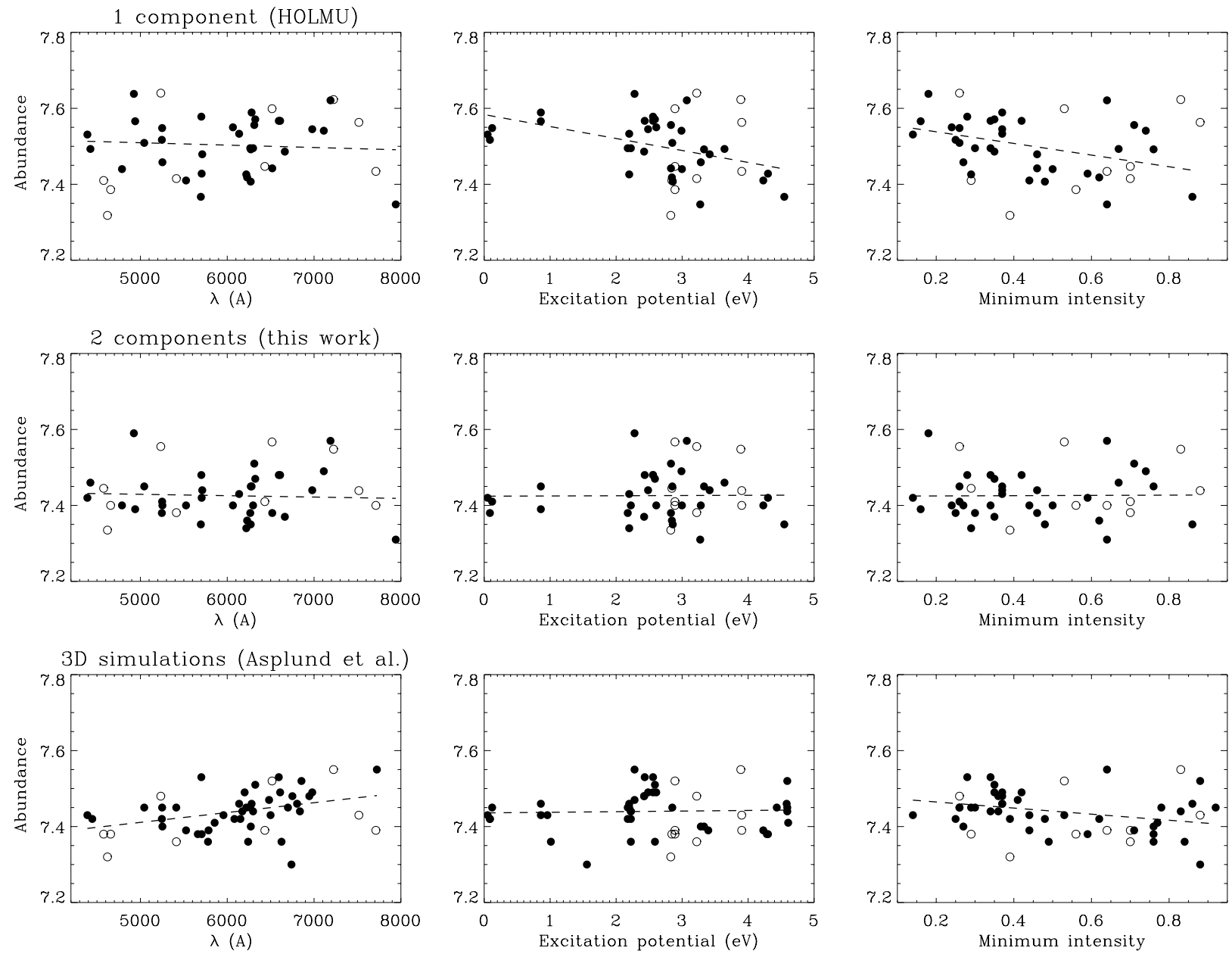

Fig. 2. Individual abundances vs. wavelength (left panels), excitation potential (central panels) and line strength (right panels) as resulting from application of HM model (first row), our two-component model atmosphere (second row), and the 3D hydrodynamical simulations of ANTS (third row). Filled circles indicate Fe I lines and open circles Fe II lines. The dashed lines represent linear least-square fits considering only the Fe I lines.

components are changed by the average temperature (defined to be $T_{\mathrm{av}}=\left[(1-\beta) T_{\mathrm{g}}^{4}+\beta T_{\mathrm{i}}^{4}\right]^{1 / 4}$, where $\beta$ is the fractional area occupied by the intergranular component), the retrieved abundances (bottom panels in Fig. 3) do not depend on either excitation potential or line strength. In this case where all temperature inhomogeneities have been removed, we find an average value of $A_{\mathrm{Fe}}=7.44 \pm 0.06$ which is almost the same as that resulting from the complete two-component model.

From these comparisons we conclude that velocity fields are an important ingredient for reliable abundance determinations. If no allowance for convective flows is made, the inferred abundances are too high by 0.04 dex on average. The physical reason is that convective motions make the line profiles to broaden, especially near the continuum where velocities are larger. If one neglects such motions and keeps the temperature fixed, the synthetic profiles are too narrow compared with the observations. In this situation, the only way to broaden the lines and hence to fit the observed profiles is to increase the abundance. As an example, we show in Fig. 4 the profiles of the Fe I line at $6978.9 \AA$ emerging from our two-component model with and without velocities, along with the observed profile. Note the larger broadening produced by velocity fields, which coincides almost perfectly with that of the observed line. As soon as microturbulence is introduced in order to compensate for the effects of the missing convective motions, the inferred abundances improve to a large extent. In particular, they no longer show any trend with excitation potential or line strength. The weaker lines are not affected by microturbulence (e.g., Gray 1992), and therefore their abundances remain the same as it is easy to verify by comparing the rightmost panels in the first and second rows of Fig. 3. By contrast, the stronger lines have their equivalent widths increased, so lower abundances are obtained. The smaller abundances of the strong lines actually remove the trends with excitation potential and line strength which appear when velocity fields are ignored. This suggests that microturbulence is an effective (but not a perfect) substitute for convective motions. Indeed, the individual abundances are too low by 0.02 dex on average $e^{1}$. The influence of temperature inhomogeneities can be assessed by considering the results

\footnotetext{
${ }^{1}$ In principle, it would be possible to obtain the same average abundance by using a slightly smaller microturbulent velocity. However, since microturbulence affects weak and strong lines in a different way, small trends with excitation potential and line strength might result.
} 

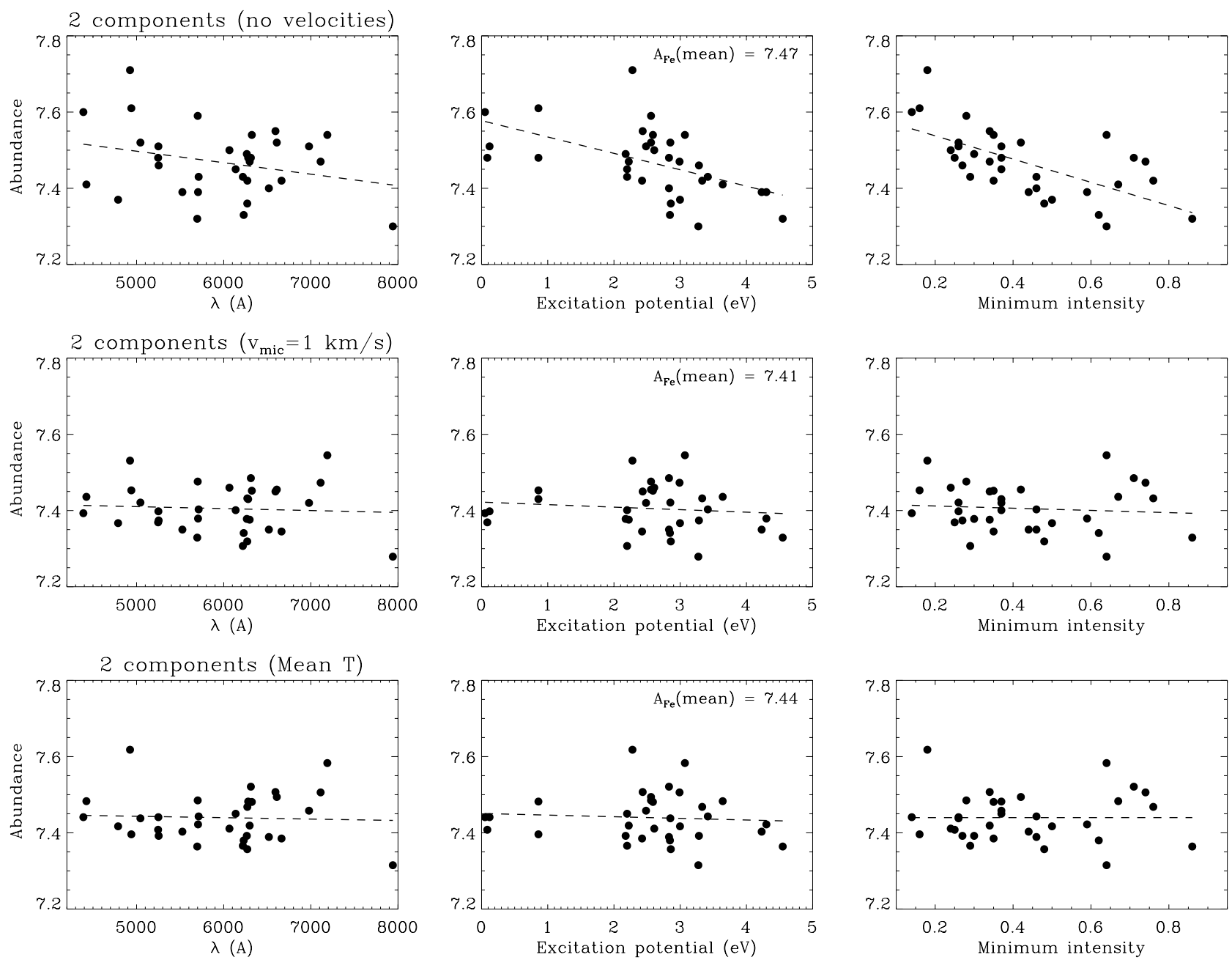

Fig. 3. Individual abundances vs. wavelength (left panels), excitation potential (central panels) and line strength (right panels) as resulting from the application of the two-component model atmosphere with the velocities set to zero (top), the velocities substituted by a microturbulent velocity of $1 \mathrm{~km} \mathrm{~s}^{-1}$ (middle), and with the original velocities but the temperatures substituted by the average stratification. Filled circles indicate Fe I lines. The dashed lines represent linear least-square fits considering all the Fe I lines.

of our last test. If the original velocity fields are kept, substitution of the granular and intergranular temperatures by the average temperature stratification makes it possible to obtain iron abundances which are in very good agreement with the values derived from the complete two-component model and the 3D hydrodynamical simulations. Keeping in mind these results, we suggest that using an appropriate thermal stratification is crucial for abundance determinations based on one-component models. In this regard, it should be mentioned that the temperature stratification resulting from our averaging the granular and intergranular temperatures is closer to the Grevesse \& Sauval (1999) model than to the HM model from $\log \tau_{5} \approx-1.5$ up to $\log \tau_{5} \approx-3.0$. Temperature stratifications retaining this feature appear to be able to mimic the effects of thermal inhomogeneities, leading to one-component abundances in good agreement with $3 \mathrm{D}$ values.

Very recently, Steffen \& Holweger (2002) have derived abundance corrections to be applied to values obtained from classical one-component models in order for them to coincide with the results of $2 \mathrm{D}$ hydrodynamical simulations.
A comparison between the 2D granular corrections of Steffen \& Holweger (2002) and our corrections $A(2 \mathrm{C})-A(1 \mathrm{C})$ is presented in Fig. 5. Consistent with the previous findings, our values (circles) and Steffen \& Holweger values (solid line) indicate that one-component model atmospheres systematically deliver larger iron abundances than models taking into account granular inhomogeneities. This abundance difference is seen to decrease with excitation potential. For Fe I lines having very small excitation potentials, classical one-component models overestimate the iron abundance by up to $0.15-0.20$ dex, while high excitation lines yield more or less the same abundance values. Our corrections are slightly larger than those of Steffen \& Holweger (2002), but the agreement is very satisfactory in view of the rather different methods employed to determine them. In particular, the 2D granular corrections refer to fictitious lines at $5500 \AA$ with excitation potentials from 0 to $6 \mathrm{eV}$. These lines are much weaker than ours (the strongest Fe I line has an equivalent width of $13 \mathrm{~m} \AA$ ). In addition, Steffen \& Holweger analysis is based on equivalent widths, whereas we fit the full profile shapes. Any of these differences 


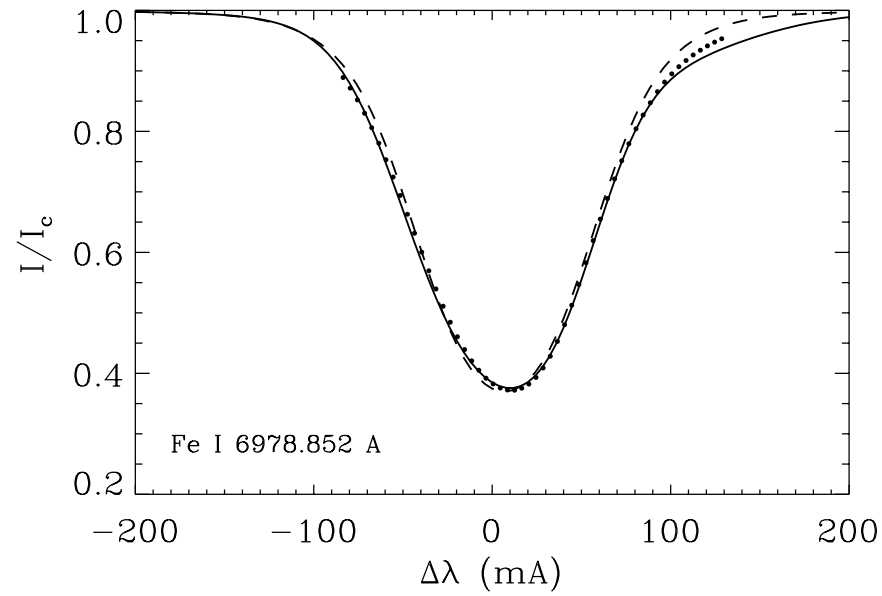

Fig. 4. Synthetic profiles of the Fe I line at $6978.8 \AA$ emerging from the two-component model of Borrero \& Bellot Rubio (2002) with (solid) and without (dashed) velocities, compared with the observed profile (dots). The iron abundance is taken to be 7.46 dex. Note the larger broadening of the profile computed with velocities. The equivalent widths are 81.0 and $75.7 \mathrm{~m} \AA$ with and without velocities, respectively.

may lead to slightly different corrections, so the important result is the overall agreement between the outcome of the 2D simulations and our two-component model.

\section{Summary}

We have determined the photospheric iron abundance by fitting the intensity profiles of $43 \mathrm{Fe}$ I and Fe II lines of the solar spectrum with the two-component model atmosphere of Borrero \& Bellot Rubio (2002). The model is able to describe the effects of convective motions in the solar photosphere, and therefore the line asymmetries are reproduced. The mean abundance resulting from $\mathrm{Fe}$ I lines is $A_{\mathrm{Fe}}=7.43 \pm 0.06$, while we estimate $A_{\mathrm{Fe}}=7.45 \pm 0.08$ from Fe II lines. Our values are in excellent accord with those derived from realistic 3D hydrodynamical simulations of the solar granulation (Asplund et al. 2000), and favor a low photospheric iron abundance. The effects of convective motions and granular temperatures have been investigated. We find that convective motions can be substituted by a microturbulent velocity of about $1 \mathrm{~km} \mathrm{~s}^{-1}$, whereas proper account of temperature inhomogeneities is achieved by adopting an average temperature stratification which is cooler than the Holweger \& Müller model in the upper layers. Although these substitutes appear to be adequate for abundance determinations based on one-component model atmospheres, it is clear that they have some limitations. From this we conclude that the use of more realistic two-component models is warranted in future analyses. Finally, it is important to mention that abundances derived from Fe I lines assuming LTE are expected to be slightly smaller than NLTE abundances (cf. Shchukina \& Trujillo Bueno 2001). Therefore, consideration of NLTE effects would probably increase the iron abundances reported here, which would then become closer to the meteoritic value.

Acknowledgements. We thank the referees, Drs. J. Sauval and N. Grevesse, for comments and suggestions which led to a significant improvement of an earlier version of the paper.

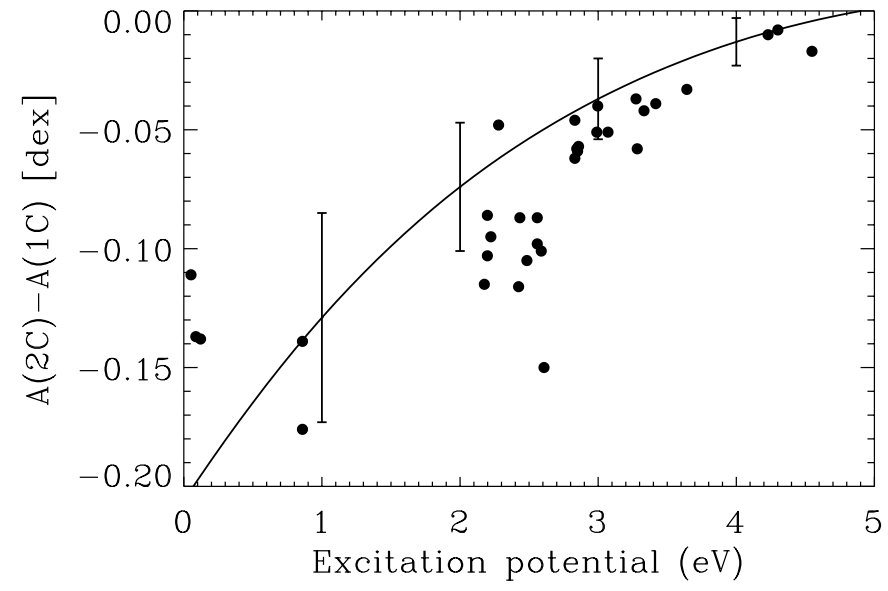

Fig. 5. Corrections to abundances determined from one-component model atmospheres as resulting from the application of our twocomponent model atmosphere (circles) and the 2D hydrodynamical simulations of Steffen \& Holweger (2002, solid line), as a function of excitation energy. The error bars indicate the statistical uncertainty of the abundance corrections obtained by Steffen \& Holweger.

\section{References}

Allende Prieto, C., \& García López, R. 1997, A\&AS, 129, 41

Allende Prieto, C., Barklem, P. S., Asplund, M., \& Ruiz Cobo, B. 2001, ApJ, 558, 830

Anstee, S. D., O’Mara, B., \& Ross, J. E. 1997, MNRAS, 284, 202

Asplund, M., Nordlund, A., Trampedach, R., \& Stein, R. F. 2000, A\&A, 359, 743 (ANTS)

Bard, A., \& Kock, M. 1994, A\&A, 282, 1014

Bard, A., Kock, A., \& Kock, M. 1991, A\&A, 248, 315

Barklem, P. S., Piskunov, N., \& O’Mara, B. J. 2000, A\&AS, 142, 467

Blackwell, D. E., Petford, A. D., \& Simmons, G. J. 1982, MNRAS, 201, 595

Blackwell, D. E., Lynas-Gray, A. E., \& Smith, G. 1995a, A\&A, 296, 217

Blackwell, D. E., Smith, G., \& Lynas-Gray, A. E. 1995b, A\&A, 303, 575

Borrero, J. M., \& Bellot Rubio, L. R. 2002, A\&A, 385, 1056

Brault, J., \& Neckel, H. 1987, Spectral Atlas of Solar Absolute Disk-Averaged and Disk-Center Intensity from 3290 to $12510 \AA$, unpublished

Frutiger, C., Solanki, S. K., Fligge, M., \& Bruls, J. H. 2000, A\&A, 358,1109

Gray, D. F. 1992, The observation and analysis of stellar photospheres, 2nd edition (Cambridge University Press)

Grevesse, N., \& Sauval, A. J. 1999, A\&A, 347, 348

Holweger, H., \& Müller, E. A. 1974, Sol. Phys., 39, 19

Holweger, H., Kock, M., \& Bard, A. 1995, A\&A, 296, 233

Kupka, F., Piskunov, N., Ryabchikova, T. A., Stempels, H. C., \& Weiss, W. W. 1999, A\&AS, 138, 119

Nave, G., Johansson, S., Learner, R. C. M., Thorne, A. P., \& Brault, J. W. 1994, ApJS, 94, 221

Neckel, H. 1994, in IAU Coll. 143, 37

Ruiz Cobo, B., \& Del Toro Iniesta, J. C. 1992, ApJ, 398, 375

Schnabel, R., Kock, M., \& Holweger, H. 1999, A\&A, 342, 610

Shchukina, N., \& Trujillo Bueno, J. 2001, ApJ, 550, 970

Steffen, M., \& Holweger, H. 2002, A\&A, 387, 258

Wittmann, A. 1974, Sol. Phys., 35, 11 